\title{
CHEMICAL COMPOSITION OF PLANETARY NEBULAE: A NEW DETERMINATION
}

\author{
V.V. GOLOVATY \\ Astronomical Observatory of Lviv University, 290005 Lomonosov str. 8, Lviv, Ukraine \\ and \\ YU. F. MALKOV \\ Crimean Astrophysical Observatory, 334413 Nauchny, Crimea, Ukraine
}

A new method of the determination of planetary nebulae abundances is proposed. Unobserved ionization stages are taken into account with aid of the correlations between relative abundances of various ions which had been obtained from the grid of the photoionization models of planetary nebulae luminescence calculated by us. Simple approximative expressions for the determination of $\mathrm{He} / \mathrm{H}, \mathrm{C} / \mathrm{H}, \mathrm{N} / \mathrm{H}$, $\mathrm{O} / \mathrm{H}, \mathrm{Ne} / \mathrm{H}, \mathrm{Mg} / \mathrm{H}, \mathrm{Si} / \mathrm{H}, \mathrm{S} / \mathrm{H}$, and $\mathrm{Ar} / \mathrm{H}$ are found. The chemical composition of 130 galactic planetary nebulae is revised. The observational data were compiled from 73 papers of many authors published in 1972-1991. Our mean abundances of C, $\mathrm{N}, \mathrm{O}, \mathrm{Ne}, \mathrm{S}, \mathrm{Ar}$ are O.1-O.3 dex lower than the mean abundances of these elements found previously by other authors. Such a discrepancy may be due to an overestimation of "empirical" ionization correction factors in previous works. It is shown that the abundance of oxygen in massive stars-precursors may be reduced by 0.2 dex on average due to the $\mathrm{ON}$-cycle, but the abundance of neon remains practically unchanged. A comparative analysis of the abundances in the galactic disk, bulge and halo nebulae is carried out. We found that helium is enhanced in the galactic bulge nebulae relative to the disk ones, but the mean nitrogen abundance and mean ratio $\mathrm{N} / \mathrm{O}$ are lower in the bulge. Our data suggest that the second dredge-up did not take place in the stars-precursors of the bulge planetaries, and the helium and nitrogen enhancement in these nebulae is due to other mixing processes. The mean abundances of $\mathrm{O}, \mathrm{Ne}, \mathrm{S}, \mathrm{Ar}$ in the galactic disk and galactic bulge nebulae are quite similar, and we must conclude that the stars-precursors of the bulge planetaries had been formed during the same burst of star formation as the stars-precursors of the disk nebulae, while the halo nebulae correspond to the previous burst. 